\title{
S100A6 Gene
}

National Cancer Institute

\section{Source}

National Cancer Institute. S100A6 Gene. NCI Thesaurus. Code C99605.

This gene is involved in calcium binding. 